(20\%), pneumonitis (13\%), anemia (13\%), diarrhea (7\%), cataract $(7 \%)$, lymphopenia $(7 \%)$, thrombocytopenia (7\%), weight loss $(7 \%)$, hypokalemia $(7 \%)$. Sixteen patients are currently evaluable for response; 6 (37.5\%) with PR, 8 (50\%) SD, 2 (12.5\%) PD; ORR 33\% (4/12) in ovarian cancer and 50\% (2/ 4) in endometrial cancer. Median PFS is 6.3 months with 95\%CI $(0.7,13.8)$ months.

Conclusions Combination rucaparib and MIRV was tolerable with mostly manageable side effects and encouraging activity in this heavily pretreated population (including prior PARPi) of both endometrial and ovarian cancer.

\section{OP008/\#194 P53ABN MOLECULAR SUBTYPE ENCOMPASSES A MORPHOLOGICALLY DIVERSE SUBSET OF ENDOMETRIAL CANCERS AND IDENTIFIES THERAPEUTIC OPPORTUNITIES TO IMPROVE OUTCOMES}

${ }^{1}$ A Jamieson* ${ }^{2} E$ Thompson, ${ }^{3}$ J Huvila, ${ }^{2} S$ Leung, ${ }^{4} \mathrm{~A}$ Lum, ${ }^{5} \mathrm{~L}$ Helpman, ${ }^{6} \mathrm{~S}$ Salvador ${ }^{7} \mathrm{~J} \mathrm{Inving,}{ }^{8} \mathrm{~K}$ Grondin, ${ }^{9} \mathrm{~A}$ Lytwyn, ${ }^{10} \mathrm{C}$ Parra-Herran, ${ }^{11} \mathrm{~S}$ offman, ${ }^{12} \mathrm{M}$ Kinloch, ${ }^{13} \mathrm{M}$ Plante, ${ }^{14} \mathrm{D}$ Vicus, ${ }^{4} \mathrm{~A}$ Talhouk, ${ }^{15} \mathrm{~S}$ Scott, ${ }^{4} \mathrm{D}$ Huntsman, ${ }^{16} \mathrm{C}$ Gilks, ${ }^{1} \mathrm{~J}$ Mcalpine. ${ }^{1}$ University of British Columbia, Gynecologic Oncology, Vancouver, Canada; ${ }^{2}$ Molecular Oncology, University of British Columbia, Vancouver, Canada; ${ }^{3}$ University of Turku, Pathology, Turku, Finland; ${ }^{4}$ University of British Columbia, Molecular Oncology, Vancouver, Canada; ${ }^{5}$ McMaster University, Gynecologic Oncology, Hamilton, Canada; ${ }^{6}$ McGill University, Gynecologic Oncology, Montreal, Canada; 'University of British Columbia, Pathology, Victoria, Canada; ${ }^{8}$ Laval University, Pathology, Quebec City, Canada; ${ }^{9}$ McMaster University, Pathology, Hamilton, Canada; ${ }^{10}$ University of Toronto, Pathology, Toronto, Canada; ${ }^{11}$ Dalhousie University, Pathology, Halifax, Canada; ${ }^{12}$ University of Saskatchewan, Pathology, Saskatoon, Canada; ${ }^{13}$ Laval University, Gynecologic Oncology, Quebec City, Canada; ${ }^{14}$ University of Toronto, Gynecologic Oncology, Toronto, Canada; ${ }^{15}$ Dalhousie University, Gynecologic Oncology, Halifax, Canada; ${ }^{16}$ University of British Columbia, Pathology, Vancouver, Canada

\subsection{6/ijgc-2021-IGCS.25}

Objectives Molecular classification of endometrial cancer (EC) has important prognostic and therapeutic implications. p53abn EC represent the most aggressive molecular subtype, and recent data has shown a survival benefit from chemotherapy and targeted therapies. We describe the clinicopathologic diversity in presentation and outcomes of p53abn ECs.

Methods Molecular classification was performed on ECs diagnosed in 2016 from 30 Canadian centres. Clinicopathologic and outcome data were collected.

Results 190 ECs were p53abn subtype; 100 serous, 33 endometrioid, 29 carcinosarcomas, 20 mixed, 6 clear cell carcinoma, 2 undifferentiated. 13 p53abn endometrioid ECs were low grade $(\mathrm{Gr} 1 / 2)$. There was a trend for worse outcomes

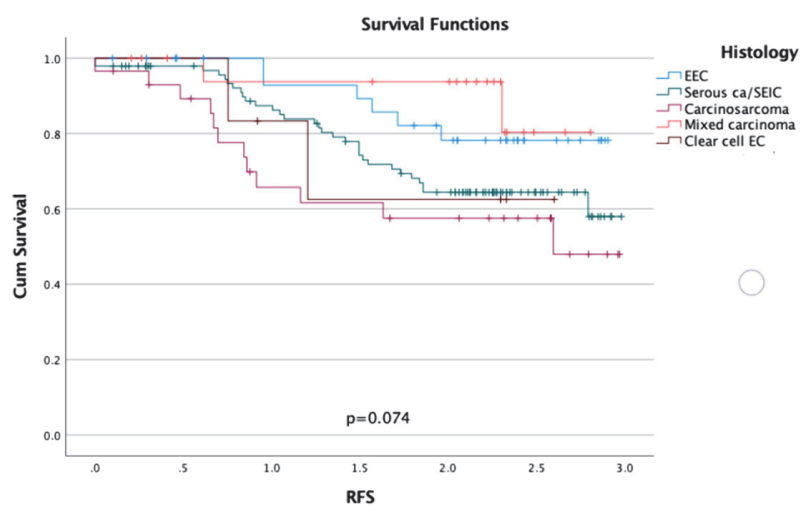

Abstract OP008/\#194 Figure 1 with non-endometrioid histotypes $(\mathrm{p}=0.074)$. Non-endometrioid p53abn ECs were more likely to present with advanced stage (III-IV) disease compared to endometrioid p53abn ECs $(43 \%$ vs $15 \%, \mathrm{p}=0.003)$. There was significant variation in adjuvant treatment; $28 \%$ patients received no adjuvant therapy, $40 \%$ received no chemotherapy. of the patients who had no chemotherapy, 20/76 (26.3\%) had a disease related event (progression/disease specific death). Stage I p53abn low grade ECs had worse outcomes (5-fold) than stage I low grade ECs of all other molecular subtypes combined.

Conclusions p53abn EC was observed across a range of histotypes including low grade ECs, with no significant difference in outcomes based on histotype. EC risk stratification based on histotype and stage failed to identify $25 \%$ of patients as high risk and $15 \%$ as intermediate risk based on the 2020 ESGO/ESTRO/ESP EC guidelines, which resulted in a missed opportunity for chemotherapy and targeted therapy.

\section{OP009/\#222 COMBINED ORAL MEGESTROL ACETATE/ LEVONORGESTREL-INTRAUTERINE SYSTEM FOR ATYPICAL ENDOMETRIAL HYPERPLASIA: A SINGLE-CENTER PROSPECTIVE RANDOMIZED CONTROLLED PILOT STUDY}

Z Xu*, B Yang, J Guan, W Shan, J Liao, Y Shi, C Ning, X Luo, X Chen. Obstetrics and Gynecology Hospital of Fudan University, Department of Gynecology, Shanghai, China

\subsection{6/ijgc-2021-IGCS.26}

Objectives To assess if addition of levonorgestrel-intrauterine system (LNG-IUS) to megestrol acetate (MA) could improve treatment outcomes for patients with atypical endometrial hyperplasia $(\mathrm{AEH})$.

Methods In this open-label randomized controlled pilot study, patients were recruited from the Obstetrics and Gynecology Hospital, Fudan University.Between June, 2017, and June, 2020, $180 \mathrm{AEH}$ patients met inclusion criteria and were randomly assigned (1:1:1) to MA+LNG-IUS group (160mg oral MA daily with LNG-IUS), LNG-IUS group or MA group (160mg oral MA daily). Hysteroscopic pathological evaluation was performed every 3 months during the treatment duration. The primary outcome, time to complete response (CR), was time from treatment initiation to pathologic assessments without lesions. Efficacy and safety were assessed in patients who received treatment. ClinicalTrials.gov: NCT03241888.

Results Median age was 33 years (range 19-44). 58 received MA, 59 received LNG-IUS and 54 received MA+LNG-IUS. At data cutoff of the analysis on January 31, 2021, median follow-up was 25.9 months (range, 2.8-43.5). CR time was significantly shorter with LNG-IUS compared with MA (median, 4.4 vs. 7.0 months; hazard ratio 1.53 ; 95\% confidence interval, $1.05-2.25 ; \mathrm{p}=0.028)$. No significant difference in CR time was found between MA+LNG-IUS group and MA group. LNG-IUS group had lower incidence of weight gain $(p<0.001)$, abdominal pain $(p=0.036)$, insomnia $(p=0.005)$, edema face $(p=0.003)$, night sweats $(p=0.003)$ and nocturia $(p=0.002)$ than MA group. MA+LNG-IUS group had higher incidence of vaginal hemorrhage $(p=0.002)$ than MA group.

Conclusions LNG-IUS significantly improved CR time compared with that for MA, with less adverse events, and might be an alternative treatment option for AEH patients. 